Proceedings of the International School and Conference on Optics and Optical Materials, ISCOM07, Belgrade, Serbia, September 3-7, 2007

\title{
Zeeman Coherences Narrowing due to Ramsey Effects Induced by Thermal Motion of Rubidium Atoms
}

\author{
Z.D. Grujić* , M.M. Mijailović, B.M. Panić, M. Minić, \\ A.G. Kovačević, M. Obradović And B.M. Jelenković \\ Institute of Physics, Pregrevica 118, 11080 Belgrade, Serbia
}

\section{AND S. CARTALEVA}

\author{
Institute of Electronics, Bulgarian Academy of Sciences \\ blvd. Tzarigradsko Shaussee 72, 1784 Sofia, Bulgaria
}

We investigated effects of spatially separated laser fields on amplitudes and widths of electromagnetically induced transparency in M-type atomic system. We have shown narrowing of the electromagnetically induced transmission resonance and therefore enhancement of medium non-linearities that can be explained by diffusion-induced Ramsey narrowing. Depending on the polarization of two laser beams, narrow probe Hanle transmission resonance changes its sign in respect of the profile obtained when the pump laser beam is off.

PACS numbers: 42.50.Gy, 32.70.Jz

\section{Introduction}

Coherence population trapping (CPT) [1], phenomena based on atomic coherence, have been intensively investigated over the past decade, primarily due to numerous applications of its manifestations. Electromagnetically induced transparency (EIT) [2] is CPT manifestation observed as characteristic, sharp dip in a laser absorption spectrum. Atomic systems which allow observation of EIT is $\Lambda$ scheme.

In most of experiments on CPT, laser beams are well overlapped to ensure good coupling of an atom with both laser fields. Typically, a buffer gas is added to alkali atoms in the cell to (a) slow motion of alkali atoms and lengthen interaction time between atom and the laser light and to (b) pressure broaden the electronic excited state which allows lasers to interact with more atoms, despite the Doppler broadening. Most models that deal with EIT assume that atom enters the laser beam with homogeneous distribution of populations among ground state levels, and that decay of the coherence is defined by the atom transit time through the

${ }^{*}$ corresponding author 
laser beam. Much less is done in terms of investigations of diffusion of coherence in gas cells, i.e., probing the coherence generated in the region which is spatially separated from the place where probing takes place.

Time and space dependent diffusion of coherently prepared atoms, so-called optical Ramsey fringes in time and frequency domain were studied by Zibrov and Matsko [3]. Also, Novikova et al. [4] show narrowing of the EIT resonance due to contribution of $\mathrm{Rb}$ atoms diffusing first out and then back to the laser beam due to collisions with a buffer gas. Recently, experiment and model were presented showing diffusion induced narrow Ramsey fringes in a time domain [5].

This work looks into effects of two separated oscillatory fields, with the same or orthogonal linear polarization, on the line widths and amplitudes of EIT. Generally, we allow pump laser beam to create atomic alignment or coherence, which then evolves in the "dark region" before atoms diffuse into the spatially separated probe laser beam. This study addresses important issues of electromagnetically induced transparency and absorption and also of stored light [6] in alkali vapors at room temperature.

\section{Experimental setup}

Experimental setup is given in Fig. 1. External cavity diode laser is locked to $F_{\mathrm{g}}=2 \rightarrow F_{\mathrm{e}}=1$ transition of ${ }^{87} \mathrm{Rb} D_{1}$ line. The laser beam is split in two

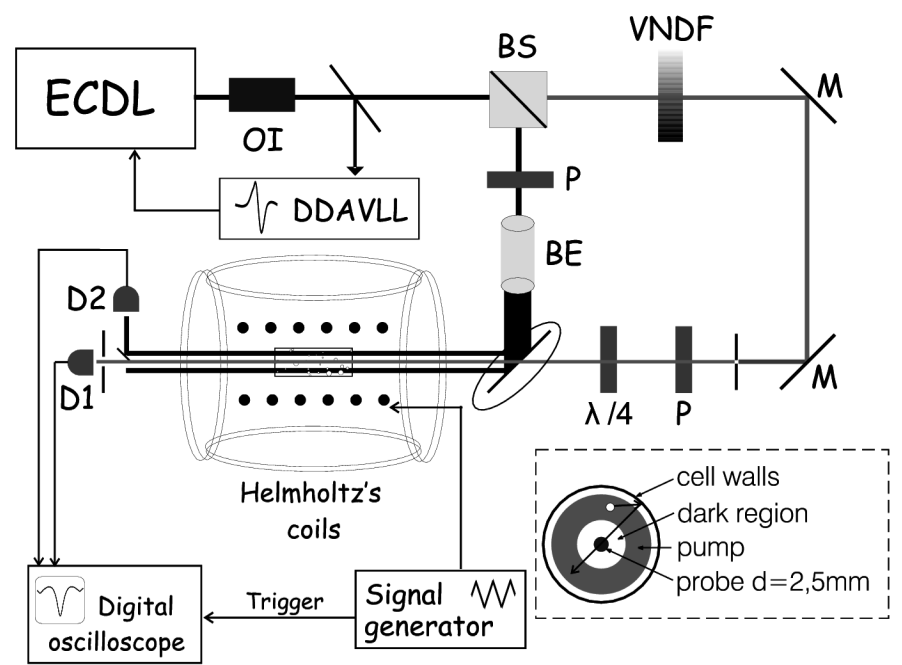

Fig. 1. Experimental setup for separated probe and pump laser beams. ECDL — diode laser, OI - optical isolator, BS - beam splitter, $\mathrm{P}$ - polarizer, $\mathrm{M}$ - mirror, VNDF — variable neutral density filter, BE - beam expander, D1 and D2 - photodetectors, DDAVLL - Doppler-free dichroic atomic vapor laser lock. Insert shows cross-section of the laser beams and the glass cell. 
parts. One beam is sent through beam expander, second through a half-wave plate. In the following text we will call the former the pump beam, and the later the probe laser beam. Two beams are recombined on the mirror with the hole at its center. The hole's diameter is $5 \mathrm{~mm}$ and $2.5 \mathrm{~mm}$ diameter probe beam passes the mirror through the hole and enters the Rb cell along its axis. As given in the inset in Fig. 1, probe and pump beam cross-sections are two concentric circles with the inner diameter of the larger (pump) beam being $5 \mathrm{~mm}$. The $8 \mathrm{~cm}$ long $\mathrm{Rb}$ vacuum cell, used in the experiment, is inside the solenoid which produces variable magnetic field along the direction of the laser beams. The cell is placed at the center of 3 pairs of large Helmholmtz's coils in order to eliminate Earth magnetic field. The intensity of transmitted probe laser light is detected with photodiode (D1), while photodiode D2 measures transmission of the pump laser beam. Signals from photodiodes, and ramp voltage used to drive current source for the solenoid, were recorded by digital oscilloscope.

\section{Results}

In the following figures we present EIT line narrowing due to atoms crossing two spatially separated excitation fields. Figure 2 shows Hanle profiles for the probe beam when the laser is locked to EIT transition $F_{\mathrm{g}}=2 \rightarrow F_{\mathrm{e}}=1$, for two probe laser powers (10 and $50 \mu \mathrm{W})$. Strong narrowing of the EIT linewidths is evident when pump is turn on, and when probe and pump polarizations are lin $\|$ lin. Narrow probe resonance switches its sign when probe and pump polarizations are lin $\perp$ lin.

Probe's EIT line width, when pump beam is turned on, is much narrower than either probe or pump EIT line widths, obtained when only one of the beams is on. This is indication that the interaction of separated laser fields resembles a Ramsey separated oscillatory field experiment where evolution of the coherence between two optical fields leads to much narrower EIT. The atoms prepared by the pump in a coherent superposition of the states leave the pump beam interaction region and either hit the wall of the glass cell losing coherence, or travel to the interaction region of the probe beam. The experimental line shapes obtained with separated pump and probe beams is average of different lengths of Ramsey sequences (time interval between excitation by the probe and by the pump) which is a function of the distance between the two beams and of atomic velocity distribution function. Different time in the "dark" means different phase shift that atom acquires between two beams.

Observed effect of the probe beam Hanle resonance sign preserving or flipping, depending if we have lin $\|$ lin or lin $\perp$ lin case, can be explained through optical pumping (alignment) created by the pump and then probed by the probe beam. As depicted in Fig. 2c, atoms are pumped by the linear pump and accumulated in edged Zeeman sublevels. When electric vector of the probe beam is parallel to that of pump (lin \| lin), narrow Hanle EIT is observed since atoms 


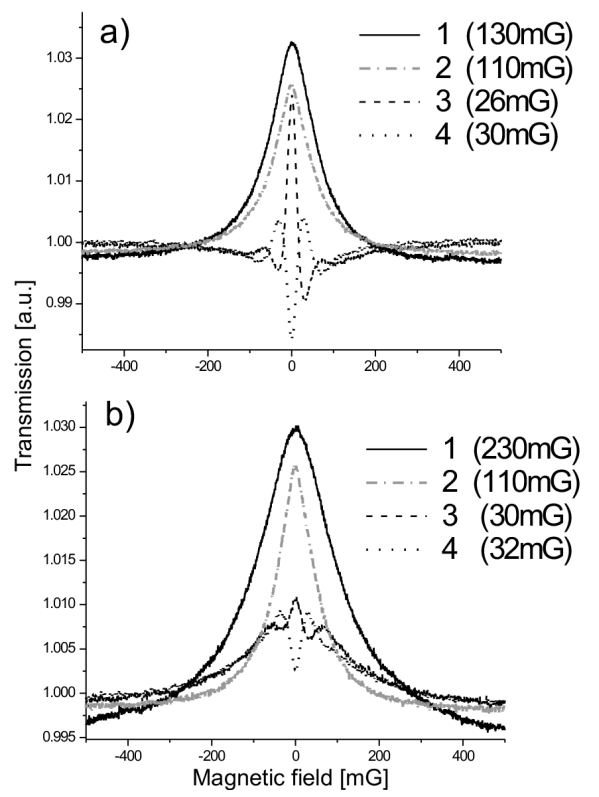

c)

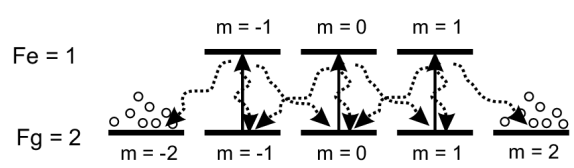

d)

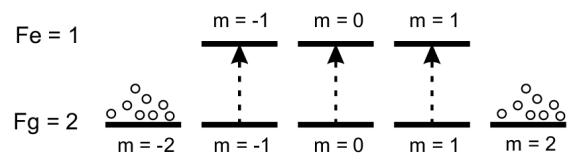

e)

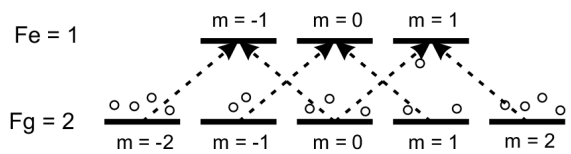

Fig. 2. Four Hanle resonances for laser locked to $F_{\mathrm{g}}=2 \rightarrow F_{\mathrm{e}}=1$ transition of ${ }^{87} \mathrm{Rb}$ $D_{1}$ line for two laser powers, $10 \mu \mathrm{W}$ (a) and $50 \mu \mathrm{W}$ (b). 1 - probe when pump is off, 2 - pump beam, 3 - probe for lin $\|$ lin case, and 4 - probe for lin $\perp$ lin case. Atomic level scheme corresponding to this transition for lin $\|$ lin (c) and (d), and for $\operatorname{lin} \perp \operatorname{lin}(\mathrm{e})$.

entering the probe region have been pumped out of Zeeman sublevels interacting most strongly with the probe laser light (Fig. 2d). When the probe beam is orthogonal to the pump beam (lin $\perp \operatorname{lin}$ ), circular $\sigma^{+}$and $\sigma^{-}$components couple most strongly those edged Zeeman sublevels which are highly populated by the pump beam (Fig. 2e), leading to absorption gain.

Typical experimental EIT resonances in both probe-pump and Hanle studies with vacuum cells have Lorentzian line shapes. Such are the results also of calculations which assume that atoms enter the laser beam with no coherence or optical pumping. While for the lower pump beam power the resonance profile is Lorentzian (Fig. 2a), for the high laser power Hanle resonance has a sharp central peak and broader profiles away from this resonance (Fig. 2b). In the later case, the pump beam is strong enough and generates detectable EIT. Due to much shorter time that atoms spend in the probe beam compared to the "dark" period, this EIT is broader and therefore contributes to wider pedestal onto which the EIT induced by the Ramsey process of separated beams is superimposed. 


\section{Acknowledgments}

The authors would like to acknowledge the support by the Ministry of Science of Republic of Serbia, under grant number 141003.

\section{References}

[1] E. Arimondo, Prog. Opt. 35, 257 (1996).

[2] G. Alzetta, L. Moi, G. Orriols, Nuovo Cimento B 52, 209 (1979).

[3] S.A. Zibrov, A. Matsko, Phys. Rev. A 65, 013814 (2001).

[4] I. Novikova, Y. Xiao, D.F. Philips, R.L. Walsworth, J. Mod. Opt. 52, 2381 (2005).

[5] Y. Xial, I. Novikova, D. Phillips, R.L. Walsworth, Phys. Rev. Lett. 96, 043601 (2006).

[6] M.D. Lukin, Rev. Mod. Phys. 75, 457 (2003). 